\title{
The Morphology Analysis of Plasma-Sprayed Cast Iron Splats at Different Substrate Temperatures via Fractal Dimension and Circularity Methods
}

https://doi.org/10.1515/htmp-2019-0021

Received August 24, 2018; accepted December 05, 2018

\begin{abstract}
Plasma-sprayed cast iron splats were deposited onto polished aluminum substrates preheated to different temperatures ranging from $25^{\circ} \mathrm{C}$ to $250^{\circ} \mathrm{C}$. The morphology of single splat was observed by a field emission scanning electron microscope. Quantitative characterization methods, including fractal dimension (FD) and circularity analyses of the splat profile, were employed to identify the difference in morphology of the splats with the change of the substrate temperature. The results showed that the substrate temperature has a significant effect on the spreading of molten droplets and the morphology of resultant splats through changing the solidification rate of the droplets. With the increment of substrate temperature, the homogeneous and sufficient spreading of the droplets resulted from low solidification rate reduces the splashing of the droplets. In addition, the evaporation of adsorbed moisture on the substrate improves the wettability between the spreading droplet and the substrate, then benefits the homogeneous spreading of the molten droplet. As a result, a distinct decline in the FD value was observed. It was also suggested that the FD analysis could be used to characterize the morphology of the splat more effectively while the circularity method was heavily dependent on the area of the splat.
\end{abstract}

Keywords: substrate temperature; splat morphology; fractal dimension; circularity

*Corresponding author: Ya-Zhe Xing, Engineering Research Center of the Ministry of Education for Pavement Materials, School of Materials Science and Engineering, Chang'an University, Xi'an, Shaanxi 710061, China, E-mail: xingyz@chd.edu.cn

Qiulan Wei, Department of Automotive Engineering, Shaanxi College of Communication Technology, Xi'an, Shaanxi 710018, China, E-mail: weiqiulan@126.com

Li He: E-mail: 690016789@qq.com,

Zhang Liu: E-mail: 1109435306@qq.com,

Xiao Feng: E-mail: 2390204966@qq.com,

Engineering Research Center of the Ministry of Education for Pavement Materials, School of Materials Science and Engineering, Chang'an University, Xi'an, Shaanxi 710061, China

\section{Introduction}

In the field of mechanical manufacturing, atmospheric plasma spray technique has been widely used to prepare metallic and ceramic coatings on mechanical parts to protect the parts surfaces from abrasion, corrosion and heat [1-4]. It is well known that the plasma-sprayed coatings are built-up by a stream of molten particles which coalesce and solidify after impacting on rough substrate or solidified coatings. It was also confirmed that the formation of a splat is nearly independent [5]. Obviously, the formation of individual splat has great effect on the properties of the coatings, and then affects the lifetime of machinery parts.

As reported up to now, there are lots of factors, including the spray conditions $[6,7]$, the spray materials [8], the substrate oxidation $[6,8]$, the roughness $[9-11]$ of the substrate surface and the wetting of droplet to substrate surface $[6,8]$, that could influence the splat formation. Small changes in these factors may lead to the large differences in the morphology of the splats, the splatsubstrate bonding, the microstructure of the coating and the residual stress in the coating [12,13], and then dramatically influence the properties of the obtained coatings. Our previous work [14] showed that the metallurgical bond between the cast iron splat and the aluminum substrate appeared when the initial substrate temperature exceeded $190^{\circ} \mathrm{C}$. Yang etal. $[15,16]$ investigated the influence of ambient pressure on splat formation. They found that the splat shape changed to the disk-like shape with the reduction of the ambient pressure, resulting in a significant increase in the adhesion strength. Sakakibara et al. [17] studied the plasma-sprayed yttria-stabilized zirconia particles on a polished stainless steel surface and confirmed that the transition to disk-like splats occurred at a substrate temperature range of $150-200^{\circ} \mathrm{C}$. When the temperature of the substrate exceeds this transition temperature, the coating consists of a large proportion of disk-like splats and shows a significant increase in adhesion strength. Generally, the splat forms two morphologies during spraying, one presents a disk-like shape and the other behaves as a splashed splat which deteriorates the quality of the 
coating. In general, the splashing occurs during the spreading of a droplet even on a flat surface at ambient atmosphere, leading to the formation of an irregular splat [8]. Many studies have been done on the correlation between the properties of the coating and the shape of the splats [18-21]. All these works suggest that the occurrence of the splashed splats goes against the formation of the good splat-substrate or inter-splat bonding, as well as the adhesion or cohesion strength. The splat-substrate adhesion determines the adhesive strength of the coating, while the inter-splat interface bonding dominates the cohesion strength of the coating. Thereby, if all the firstlayer splats adhere to the substrate and the inter-splat bonding is excellent, then the enhancements in both the adhesion strength and the cohesion strength can be expected. On the contrary, the poor adhesion between the splat and the substrate produces when the splashed splats form on the surface of the substrate, because the positions where the splash fingers formed will act as the weak bonding sites [15]. Moreover, the weak bonding sites between the splats lead to a decreased cohesion of the coating by weakening the inter-splat bonding.

In conclusion, the morphology of the splats affects the microstructure of the coating, and then affects the performance of the coating. Therefore, it is significant to employ some feature parameters to quantitatively characterize the morphology of the splat. These parameters can establish some quantitative relationships, such as the relationship between the spray parameters and the splat morphology and the relationship between the splat morphology and the properties of the coating. That is to say, a quantitative description of the splat morphology can contribute to establishing a quantitative relationship between the spray parameters and the coating properties. Chen et al. [22] employed the image analysis method to identify the splat in the field emission scanning electron microscope (FESEM) image. The fractal dimension (FD) and the solidity were used to quantitatively characterize the morphology of the splat. Kang et al. [23] defined the aspect ratio and the fractional factor to investigate the influence of the impacting angle on the spreading and the solidification behaviors of a molten droplet. The results showed that both the aspect ratio and the fractional factor increase with the increase in the inclination angle of the substrate. Mulero et al. [24] developed an image inspection system to characterize the splat in terms of its area, perimeter, circularity, eccentricity and solidity, which can quantify the morphology of a thermally sprayed splat.

In the present work, polished aluminum substrates were preheated to different temperatures, then the cast iron splats were plasma-sprayed onto the preheated substrate surface. The morphologies of the splats were quantitatively characterized by the circularity and the FD of the splat periphery. The effect of the substrate temperature on the geometric parameters (circularity and FD) of plasma-sprayed splats was investigated.

\section{Experimental process}

\section{Materials and preparation of individual splats}

Commercially available Fe-4.1C-1.5Si-1.5B-36.6Cr (in weight percentage) powder (DG.Fe-05, Chengdu Daguang Thermal Spraying Materials Co. Ltd., China) was used as the starting powder The powder consists of spherical particles with a range in size from 20 to $75 \mu \mathrm{m}$ (as shown in Figure 1). A commercial plasma spray system (GP-80, Aerospace Research Institute of Materials \& Processing Technology, China) was employed to deposit the cast iron splats. Pure aluminum disks $(99.7 \%, 20 \mathrm{~mm}$ in diameter, $4 \mathrm{~mm}$ in thickness) were used as the substrate. Before spraying, all aluminum disks were mirror-polished until their surface roughness $(\mathrm{Ra})$ reached about $0.03 \mu \mathrm{m}$, which was measured by a surface roughmeter (TR-240, Beijing Time Group, China). The traverse speed of the plasma torch was fixed at $1,200 \mathrm{~mm} / \mathrm{s}$. The flow rate of the powder was fixed at $4 \mathrm{~L} / \mathrm{min}$. The substrate was preheated by the plasma jet without carrying powders, and the preheating temperature was monitored using a pyrometer (RAYRPM30L3U, Raytek, USA). When the temperature of the substrate surface reached a set value shown in Table 1, the powders were

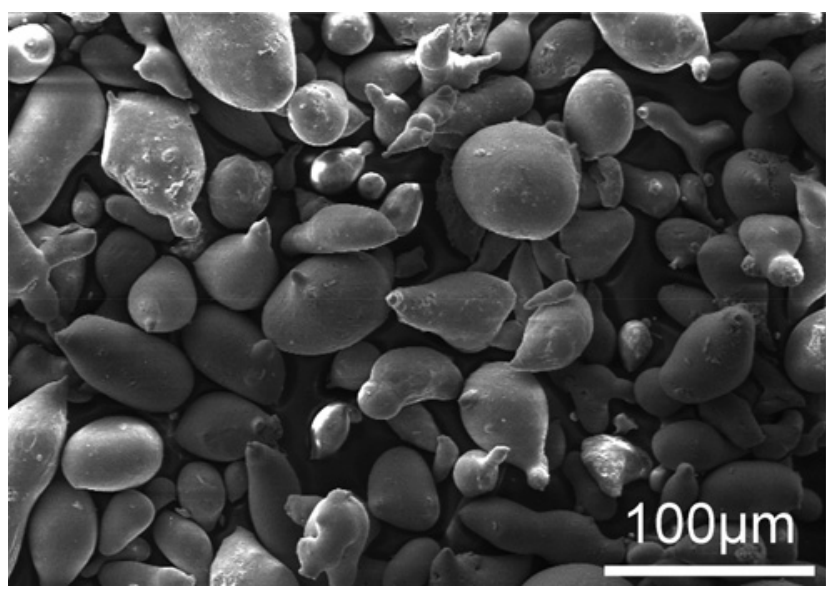

Figure 1: The morphology of the feedstock powders. 
Table 1: Spray parameters for the deposition of cast iron splats on the aluminum surface.

\begin{tabular}{lrrrrr}
\hline Sample & Arc power $(\mathrm{kW})$ & Spray distance $(\mathrm{mm})$ & \multicolumn{2}{c}{ Gas flow rate (L/min) } & Substrate temperate $\left({ }^{\circ} \mathbf{C}\right)$ \\
& & & Ar & $\mathbf{H}_{\mathbf{2}}$ & \\
\hline A & 30 & 80 & 32 & 6.3 & $25 \pm 2$ \\
B & 30 & 80 & 32 & 6.3 & $150 \pm 8$ \\
C & 30 & 80 & 32 & 6.3 & $250 \pm 10$ \\
\hline
\end{tabular}

immediately carried into plasma jet. Table 1 lists all the spray parameters for the deposition of the cast iron splats on the aluminum surface. Obviously, in addition to the difference of the substrate temperature, other preparation conditions for all samples were identical.

\section{Morphology and geometric parameters of individual splats}

After spraying, the morphology of the splats deposited under different conditions was observed by a commercial FESEM (S-4800, Hitachi, Japan). For each sample, ten images of individual splats were obtained with a resolution of 1,024 $\times 768$ pixels and 24-bits per pixel. The magnification of all selected images is $500 \times$. The calculation process of the geometric parameters of single splat contains the following steps: (1) image denoising, (2) binarization processing, (3) Canny algorithm edge detection, (4) FD calculation of the splat periphery using the boxcounting method, (5) circularity calculation of the same splat after the FD calculation. All calculations were conducted with a commercially available software, MATLAB R2014. As reported by Ding and Goshtasby [25], the object boundaries in the image can be obtained by a Canny edge detector. The Canny edge detector classifies a pixel as an edge if the gradient magnitude of the pixel is larger than those of the pixels at both its sides in the direction of the maximum intensity change. Therefore, we used the Canny edge detector to obtain the boundaries of the splat. In the process of edge detection, the splashed parts produced during the spreading of the molten droplet were removed because they were not connected to the main part of the splat.

Circularity specifies the relationship between the area and the perimeter of a splat (see eq. (1) [24]) and signifies the degree of approximation of a splat to a circle. Furthermore, area is calculated by adding the actual number of pixels in the region. Perimeter is defined as the distance around the periphery of the region, and it is measured by calculating the distance between each adjoining pair of the pixels around the border of the region.

$$
\text { Circularity }=\frac{4 \pi \cdot \text { Area }^{2}}{\text { Perimeter }^{2}}
$$

The FD was utilized to quantitatively describe the complexity of the periphery of a splat. A box-counting method was employed to evaluate the FD. In the process of the box-counting method, a series of squares were used to cover the profile of a splat. The relationship between the square size $\varepsilon$ and the number of the squares $N(\varepsilon)$ can be expressed as [26]:

$$
N(\varepsilon) \propto \varepsilon^{-D}
$$

where $D$ is the FD of the periphery of a splat, which can be calculated by the linear regression given as [26]:

$$
\log N(\varepsilon)=-D \log \varepsilon+C
$$

\section{Results and discussion}

\section{Effect of substrate temperature on geometric parameters of the splats}

Figure 2 shows the changes in the FD and the circularity of the splats. With the increment of the substrate temperature, the FD decreased and the circularity increased. When the substrate temperature was $25 \pm 2^{\circ} \mathrm{C}$, the FD was $1.3201 \pm 0.0359$ and the circularity was $0.3001 \pm 0.0880$. As the substrate temperature increased from $25 \pm 2^{\circ} \mathrm{C}$ to $150 \pm 8^{\circ} \mathrm{C}$, the FD decreased to $1.2348 \pm 0.0509$ and the circularity increased to $0.5693 \pm 0.1493$. These changes in the FD and the circularity can be reasonably explained by the evaporated gas-induced splashing model [27]. The substrate surface was easy to be covered by the adsorbates when the substrate temperature remained at room 


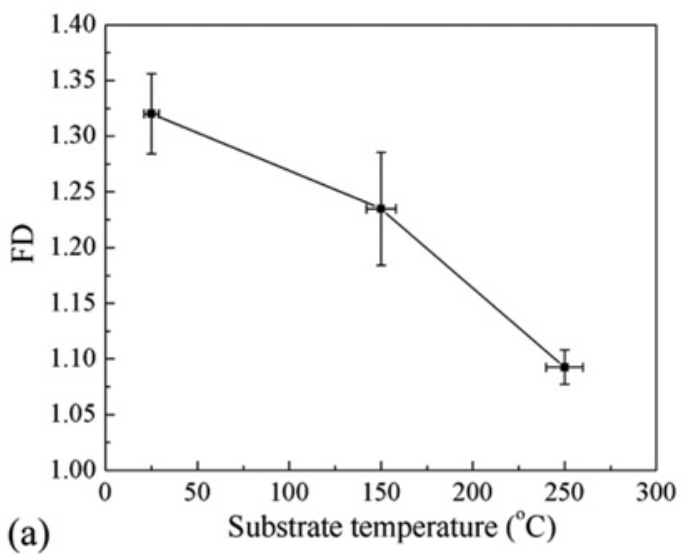

(a)

Figure 2: The changes in the FD (a) and the circularity (b) of the splats with the substrate temperature.

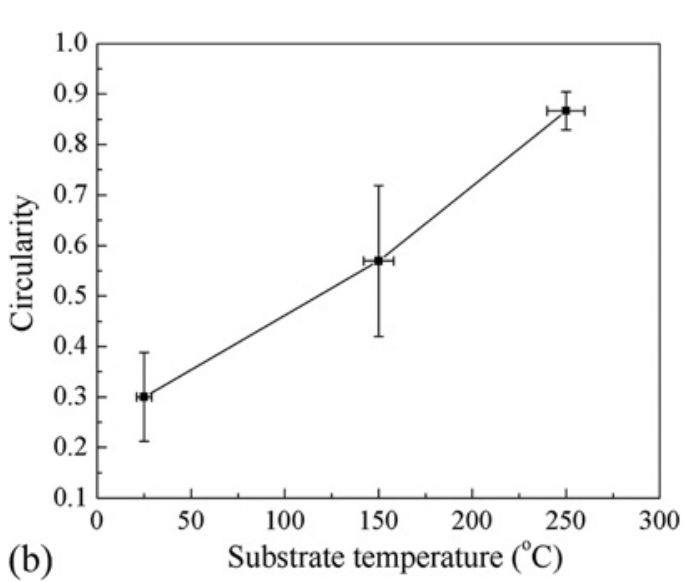

(b) temperature. During the deposition process of the droplet, the substrate surface was heated rapidly by the impacting droplets. As a result, the adsorbates gasified and evaporated from the substrate surface. Then, the air flow generated by the evaporation gas induced the splashing, which increased the complexity of the splat periphery (see Figure 3(a)). As a result, the FD reached the maximum value. As mentioned in the "Morphology and geometric parameters of individual splats" section, the circularity represents the degree of approximation of a splat to a circle. However, a splat is close to a circle, indicating the homogeneous spreading of the molten droplet. Uniform spreading of a molten droplet reduces the complexity of the periphery of the resultant splat, leading to the reduction in the FD value. When the substrate was preheated to $150 \pm 8^{\circ} \mathrm{C}$, the adsorbates on the substrate surface were evaporated and removed. Consequently, the wettability of the molten droplet on the substrate surface was improved [28], resulting in a relatively uniform spreading of the droplet and the formation of a splat with restrained splash (see Figure 3(b)). This eventually caused the decrease in the FD and the increase in the circularity of the splats. Interestingly, at this temperature $\left(150 \pm 8^{\circ} \mathrm{C}\right)$, the ranges of the values in the FD and the circularity were much greater than those of the samples prepared at room temperature and $250 \pm 10^{\circ} \mathrm{C}$. In accordance with the previous report [27], when the substrate was preheated to a temperature above the boiling point of the adsorbates, the obtained splat presented a disk shape. In the present work, all samples were polished in a pollution-free environment. This indicates the adsorbates on the surface of the substrate should be mainly water. That is to say, when the substrate was preheated to above $100^{\circ} \mathrm{C}$ (boiling point of water), the spreading of the droplets was more homogeneous due to the evaporation of water adsorbed on the substrate surface.

As the substrate temperature further increased to $250 \pm 10^{\circ} \mathrm{C}$, the FD decreased continuously and the circularity increased continuously. With preheating the substrate to a higher temperature, the cooling rate of the droplets decreased due to the reduced temperature difference between the droplet and the substrate and the oxidation of the substrate surface [14], delaying the solidification of the spreading droplets and then causing the homogeneous and sufficient spreading of the droplets. As a consequence, a regular disk splat was expected (see Figure 3(c)) and both the minimum FD value and the maximum circularity value were obtained in the present temperature range.

\section{Comparison of the FD and the circularity}

\section{Relationship between the FD and the circularity}

As mentioned in the "Effect of substrate temperature on geometric parameters of the splats" section, both a higher FD and a lower circularity correspond to an irregular splash splat. This indicates a potential relationship between the FD and the circularity. Therefore, we chose 30 splats (ten splats for each sample) to plot the relationship between the FD and the circularity of them, as shown in Figure 4. Meanwhile, the linear fitting of the data was also conducted. Apparently, there was a negative linear correlation between the FD and the circularity due to the high linear fitting correlation coefficient $(R=0.9772)$.

As mentioned in the "Morphology and geometric parameters of individual splats" section, the circularity 

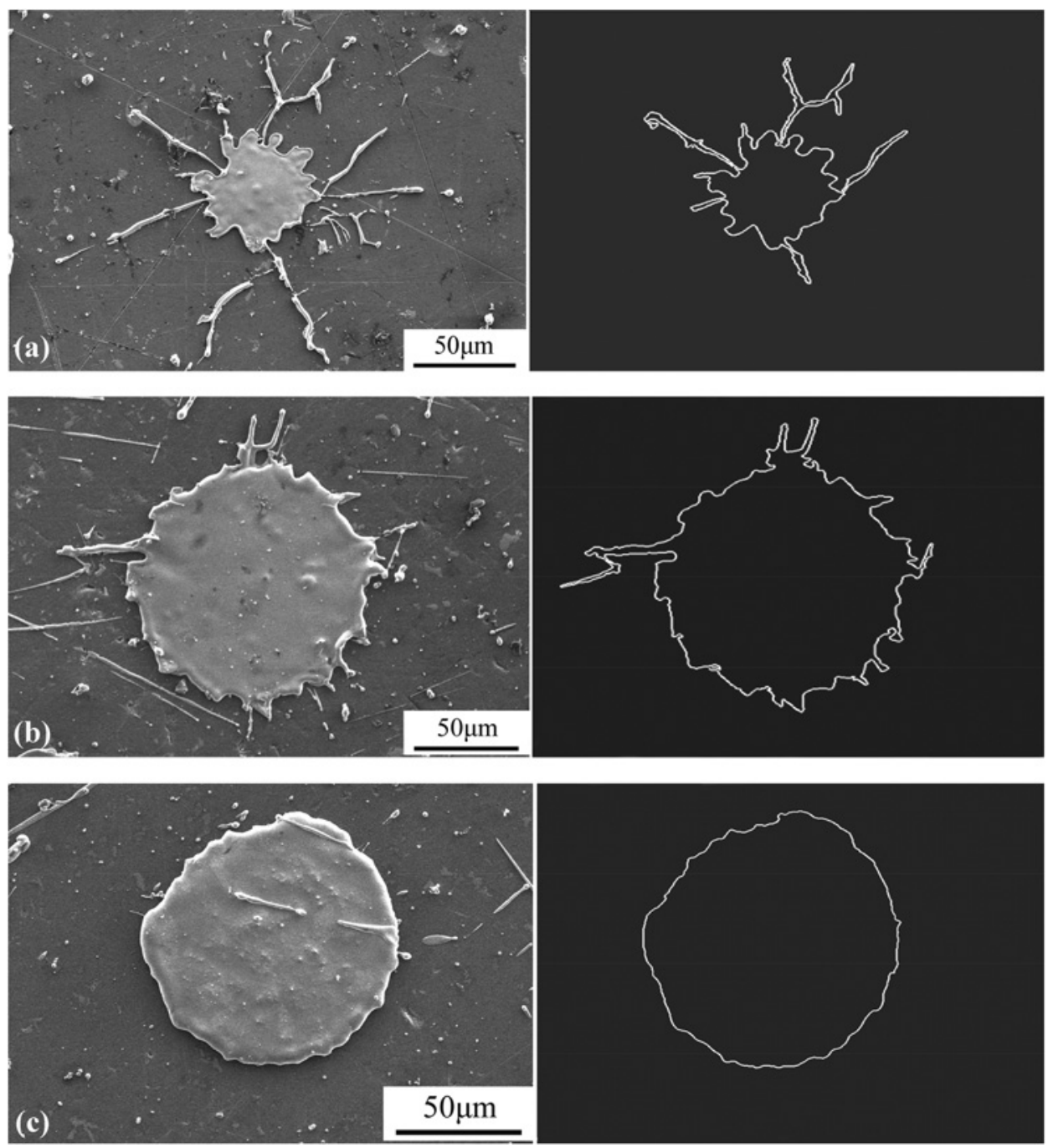

Figure 3: Typical splat deposited on the substrate preheated to (a) $25 \pm 2^{\circ} \mathrm{C}$, (b) $150 \pm 8^{\circ} \mathrm{C}$ and (c) $250 \pm 10^{\circ} \mathrm{C}$. The left micrographs are FESEM images and the right micrographs are the corresponding images after Canny algorithm edge detection.

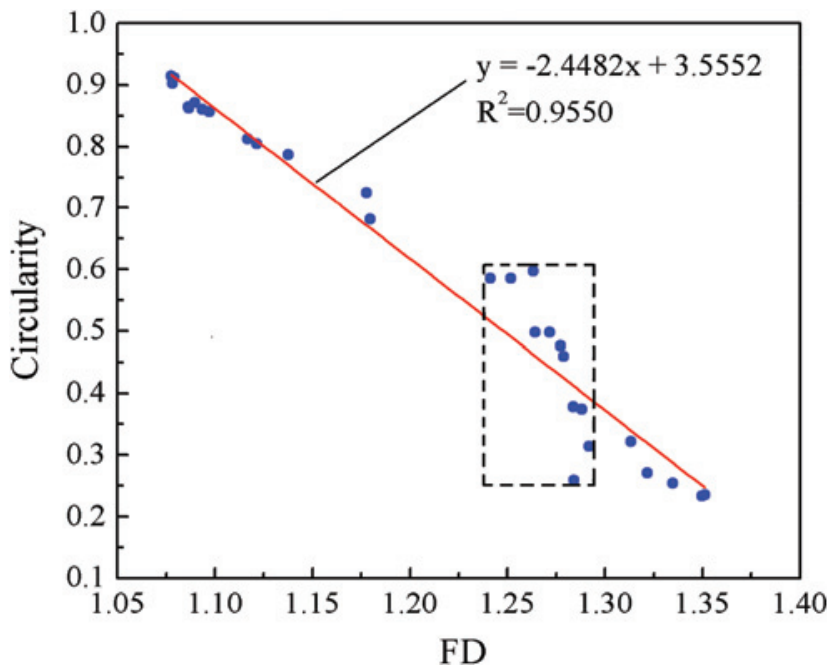

Figure 4: Relationship between the FD and the circularity of the splats. signifies the degree of approximation of a splat to a circle, and the FD describes the complexity of the periphery of a splat. Despite the difference in the concepts of two geometric parameters, a strong correlation exists between them (see Figure 4). This is related to the formation mechanism of the splats. Figure 5 shows the schematic diagrams of forming processes of two typical splats with different morphologies. In Figure 5, the arrows signify the spreading direction of the melts, and the length of the arrows represents the spreading speed. When the spreading speeds of the droplet in different directions are identical, a disk splat (see Figure 4(a)), which is close to a circle without complex periphery, is prone to form. Inversely, when the spreading speeds of the droplet in all directions are different, a splash-shaped splat (see Figure 4(b)), which possesses an irregular shape with 


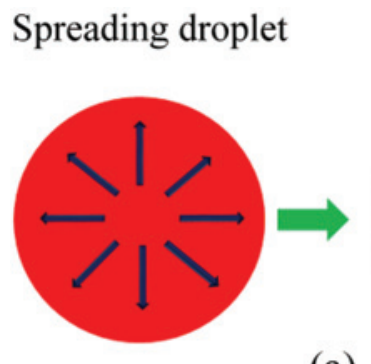

(a)
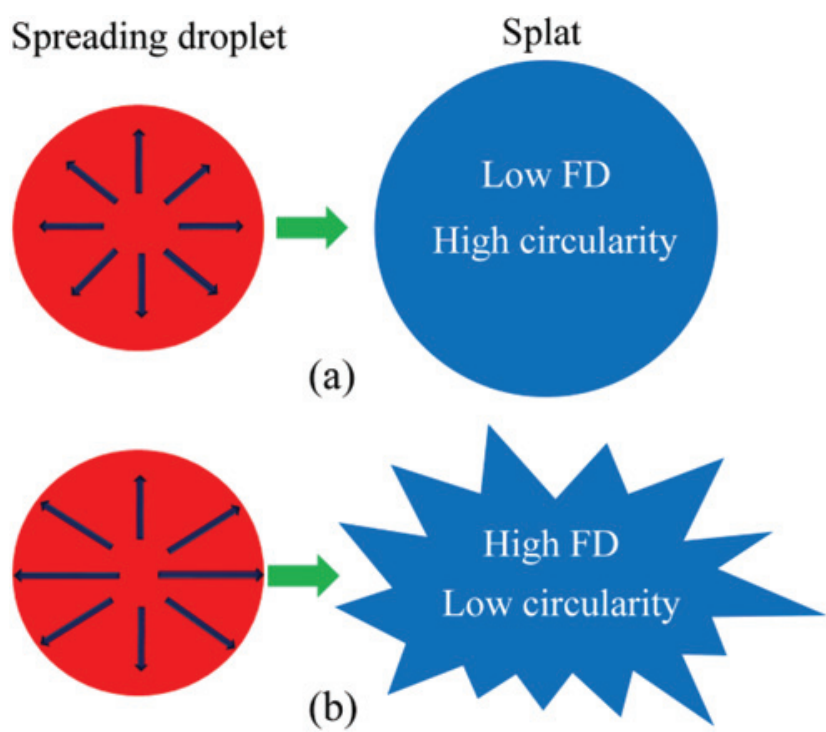

Figure 5: Schematic diagrams of the forming processes of typical morphologies of two splats. The left images present the morphologies of the splats at an early stage of spreading, while the right images present the morphologies of final splats.

complex periphery, is easy to form. That is to say, the FD and the circularity are consistent in characterizing the morphology of a plasma-sprayed splat. High circularity of a splat signifies a uniform spreading of the molten droplet, which leads to the reductions in the complexity and the FD value of the resultant splat.

\section{Applicability of the circularity}

According to the previous analyses, there was a negative correlation between the FD and the circularity (see the fitted line in Figure 4). However, some outliers in the data of individual splats were far away from the straight line. Moreover, these outliers were concentrated in a circularity range of $0.2586-0.5976$ and a FD range of 1.2412-1.2918, which were marked by the dotted square box in Figure 4. This indicates that the values in this small range do not fit the straight line. Therefore, in this range, two typical splats (see Figure 3(a) and 3(b), marked by SP1 and SP2 respectively) were selected from sample A and sample B to reveal the changes in the FD and the circularity of the splats. The corresponding values were listed in Table 2. It was found that the FD values of two splats were approximately identical, while the circularity of SP2 was about twice that of SP1. Furthermore, the area of SP2 was 3.7 times that of SP1, and the perimeter of SP2 was 1.4 times that of SP1. These indicate that the area of the splat seriously affects the circularity value. Even if the complexities of the peripheries of two splats were approximately similar, the circularity value increased significantly with the increase in the area of the splat. Generally, the area of the splat is determined by both the particle size of the starting powder and the spreading of the molten droplet. With a certain flattening ratio, thereby, the particle size of the starting powder will be the main factor affecting the area of the splat. As for the present work, the size of the powder used ranges from 20 to $75 \mu \mathrm{m}$. Such a wide range of particle size makes the final area of the splat different, leading to a wide range of the circularity value. Therefore, in order to use the circularity to characterize the morphology of the splats, a small difference in the size of the starting powders is required. The comparability in the circularity is only valid for the powders with comparable sizes. In contrast with the circularity, the FD is more suitable for characterizing the morphology of the splats with different sizes.

\section{Conclusions}

Individual cast iron splats were prepared on the mirrorpolished aluminum substrates preheated to different temperatures. The morphologies of the resultant splats were quantitatively characterized by both the FD and the circularity. With increasing the preheating temperature, the FD value of the splats decreased and the circularity value increased. For the splats deposited at room temperature, a maximum FD value, which corresponded to a complex periphery of the splats, was acquired due to the strong splashing of the droplet induced by the evaporation of the adsorbed moisture on the substrate surface. With preheating the substrate to $150 \pm 8^{\circ} \mathrm{C}$, most of the water on the substrate surface was removed at a high temperature and the wettability between the droplet and the substrate surface was improved. This caused the homogeneous spreading of the droplet and consequently led to a reduced FD value. When the substrate was further preheated to $250 \pm 10^{\circ} \mathrm{C}$, the homogeneous and sufficient spreading of the droplet caused by lower solidification rate significantly suppressed the splashing of the droplet. As a result, the FD value decreased while the circularity increased accordingly.

Compared with the circularity, the FD was more excellent in characterizing the morphology of splat. The FD and the circularity were intrinsically consistent when using them to characterize the morphology of the splat. 
Table 2: The main parameters of two splats.

\begin{tabular}{lrrrrr}
\hline Splat & Substrate temperate $\left({ }^{\circ} \mathrm{C}\right)$ & FD & Circularity & Area $\left(\mu \mathrm{m}^{2}\right)$ & Perimeter $(\mu \mathrm{m})$ \\
\hline SP1 & $25 \pm 2^{\circ} \mathrm{C}$ & 1.2843 & 0.2586 & $2,162.5104$ & 324.0847 \\
SP2 & $150 \pm 8^{\circ} \mathrm{C}$ & 1.2718 & 0.4982 & $7,923.0043$ & 446.9282 \\
\hline
\end{tabular}

However, the circularity is heavily dependent on the area of the splat. Therefore, reducing the size difference of the original powders can enhance the applicability of the circularity in characterizing the morphology of the splats.

Funding: This work was supported by the Special Scientific Research Project of Shaanxi Provincial Department of Education (18JK0081).

\section{References}

[1] R. Dhiman, A.G. Mcdonald and S. Chandra, Surf. Coat. Technol., 201 (2007) 7789-7801.

[2] J.J. Kang, B.S. Xu, H.D. Wang and C.B. Wang, Tribol. Int., 73 (2014) 47-56.

[3] Z.Y. Piao, B.S. Xu, H.D. Wang and C.H. Pu, Appl. Surf. Sci., 257 (2011) 2581-2586.

[4] L. Pawlowski, The Science and Engineering of Thermal Spray Coatings, Second edition, John Wiley \& Sons, England (2008).

[5] S. Kuroda and T.W. Clyne, Thin Solid Films, 200 (1991) 49-66.

[6] L. Blanchi, A. Grimaud, F. Blein, P. Lucchèse and P. Fauchais, J. Therm. Spray Technol., 4 (1995) 59-66.

[7] G. Montavon, S. Sampath, C.C. Berndt, H. Herman and C. Coddet, J. Therm. Spray Technol., 4 (1995) 67-74.

[8] M. Vardelle, A. Vardelle, A.C. Leger, P. Fauchais and D. Gobin, J. Therm. Spray Technol., 4 (1995) 50-58.

[9] C. Moreau, P. Gougeon and M. Lamontagne, J. Therm. Spray Technol., 4 (1995) 25-33.

[10] S. Amada, K. Imagawa and S. Aoki, Surf. Coat. Technol., 154 (2002) 27-33.

[11] M. Raessi, J. Mostaghimi and M. Bussmann, Thin Solid Films, 506-507 (2006) 133-135.
[12] K. Yang, M. Fukumoto, T. Yasui and M. Yamada, Surf. Coat. Technol., 214 (2013) 138-143.

[13] E.J. Yang, X.T. Luo, G.J. Yang, C.J. Li, M. Takahashi, S. Kuroda and K.H. Kim, Ceram. Int., 42 (2016) 853-860.

[14] Y.-Z. Xing, Z. Liu, G. Wang, X.-H. Li, C.-P. Jiang, Y.-N. Chen, Y. Zhang, X.-D. Song and M. Dargusch, Surf. Coat. Technol., 316 (2017) 190-198.

[15] K. Yang, K. Tanaka, T. Usami, M. Fukumoto, T. Yasui, M. Yamada and Q.J. Jpn, Weld. Soc., 29 (2011) 151s-155s.

[16] K. Yang, M. Liu, K. Zhou and C. Deng, J. Mater., 2013 (2013) 1-14.

[17] N. Sakakibara, H. Tsukuda and A. Notomi, The splat morphology of plasma sprayed particle and the relation to coating property, Proceedings of the International Thermal Spray Conference, ASM International, Montreal, QUE, United states (2000), pp. 753-758.

[18] P. Fauchais, A. Vardelle, M. Vardelle and M. Fukumoto, J. Therm. Spray. Technol., 13 (2004) 337-360.

[19] S. Chandra and P. Fauchais, J. Therm. Spray. Technol., 18 (2009) 148-180.

[20] J. Mostaghimi, M. Pasandideh-Fard and S. Chandra, Plasma Chem. Plasma P, 22 (2002) 59-84.

[21] M. Fukumoto, T. Yokoyama, K. Oku and Y. Tanaka, J. Jpn. High Temp. Soc., 23 (1997) 240-246.

[22] S.Y. Chen, G.Z. Ma, H.D. Wang, P.F. He, M. Liu, H.J. Wang and B.S. Xu, Appl. Surf. Sci., 409 (2017) 277-284.

[23] C.W. Kang, J.K. Tan, L. Pan, Y.L. Cheng and A. Jaffar, Appl. Surf. Sci., 257 (2011) 10363-10372.

[24] M.A. Mulero, J. Zapata, R. Vilar, V. Martínez and R. Gadow, Surf. Coat. Technol., 278 (2015) 1-11.

[25] L. Ding and A. Goshtasby, Pattern Recogn., 34 (2001) 721-725.

[26] L. Wang, Z. Wang, W. Xie and X. Song, Int. J. Fatigue, 45 (2012) 1-7.

[27] C.J. Li and J.L. Li, Surf. Coat. Technol., 184 (2004) 13-23.

[28] K. Yang, M. Fukumoto, T. Yasui and M. Yamada, J. Therm. Spray. Technol., 19 (2010) 1195-1205. 